\title{
Pharmakogenetische Tests in der ärztlichen Praxis
}

\author{
K. Faisst ${ }^{a}$, N. M. Probst-Hensch ${ }^{b}$
}

International scheint sich die Meinung durchzusetzen, dass pharmakogenetische Tests unter ärztlicher Aufsicht durchgeführt werden sollten. Dies, da die Interpretation der Testresultate oder der Entscheidungsprozess für oder gegen ein genomikbasiertes Medikament bei ausgewählten Zielgruppen Teil einer Diagnose- und Behandlungsstrategie darstellen. Die komplexen Zusammenhänge genetischer Faktoren mit nicht-genetischen Einflüssen, die bei der Entscheidungsfindung berücksichtigt werden müssen, stellen hohe Anforderungen an die beteiligten Akteure. Daher ist mit der Verbreitung von pharmakogenetischen Tests in der ärztlichen Praxis damit zu rechnen, dass der Konsultationsprozess (noch) anspruchsvoller wird. Wirksamere Behandlungen oder weniger Nebenwirkungen können aber umgekehrt auch dazu führen, dass das Vertrauen der Patientinnen und Patienten in die fachliche Kompetenz ihrer behandelnden Ärztinnen und Ärzte gestärkt wird. Dies setzt allerdings voraus, dass die Leistungserbringer über das erforderliche Fachwissen verfügen und in der Lage sind, die immer komplexer werdenden Zusammenhänge von Einflussfaktoren auf einen Therapieentscheid patientengerecht zu vermitteln.

a Institut für Sozial- und Präventivmedizin der Universität Zürich.

b Molekulare Epidemiologie/Krebsregister, ISPM Zürich/Pathologie der Universität Zürich.

Korrespondenz:

Dr. med. Karin Faisst, MPH MAE Institut für Sozial-

und Präventivmedizin

Universität Zürich

Sumatrastrasse 30

CH-8006 Zürich

Tel. 016344600

Fax 016344962

E-Mail: karin@ifspm.unizh.ch

\section{Einleitung}

Der Wunsch nach gemeinsamer Entscheidungsfindung bzw. Patientenautonomie hat in den letzten zehn Jahren deutlich zugenommen. Patientenempowerment, das heisst Partizipation und Information, wird auch künftig ein immer wichtigerer Aspekt im Arzt-Patienten-Verhältnis. Studien zeigen, dass die grosse Mehrheit der schweizerischen Bevölkerung - 91\% eines telefonisch befragten Kollektivs - wünscht, in therapeutische Behandlungsentscheide einbezogen zu werden. Hausärztinnen und Hausärzte spielen hier eine wichtige Rolle. Sie werden von den Patientinnen und Patienten als die vertrauenswürdigste und als gut zugängliche Quelle für individuell zugeschnittene Gesundheitsinformationen geschätzt [1].

Die Art und Weise, wie nun pharmakogenetische Tests den medizinischen Alltag beeinflussen werden, wird unter anderem davon abhängen, wie und von wem pharmakogenetische Tests verteilt und angeboten werden (Internet, Apotheke, Arzt). Zudem wird der praktische Umgang mit Testempfehlungen und -interpretationen eine wichtige Rolle spielen. Die folgenden Ausführungen fokussieren auf neue Herausforderungen, welche mit der breiten Einführung von pharmakogenomikbasierten Tests und Me- dikamenten auf das praktische Umfeld, in dem Medikamente verschrieben und angewendet werden, zu erwarten sind.

\section{Testanbieter}

International scheint sich die Meinung durchzusetzen, dass genetische Tests wegen der Komplexität der Resultatsinterpretation - im Gegensatz zum Beispiel zu einem Schwangerschaftstest - nicht von Patientinnen und Patienten selbständig erworben und durchgeführt werden sollten. $\mathrm{Zu}$ diesem Schluss kam kürzlich «The Human Genetics Commission» in England [2]. Für diesen Standpunkt sprechen zudem die folgenden Überlegungen:

- Es ist davon auszugehen, dass pharmakogenetische Tests und Medikamente zunächst in erster Linie für schwere Krankheiten - zum Beispiel Krebsbehandlung - und Nebenwirkungen von verschreibungspflichtigen Medikamenten - zum Beispiel Antidepressiva entwickelt werden. Die Behandlungen dieser Krankheiten dürften auch künftig unter ärztlicher Aufsicht erfolgen. Daher liegt es nahe, dass auch der Einsatz von pharmakogenetischen Tests und Medikamenten in der Verantwortung der behandelnden Ärztinnen und Ärzte liegt.

- Noch gewichtiger dürfte aber das Argument sein, dass pharmakogenetische Tests als Teil einer Diagnose- und Behandlungsstrategie zu sehen sind. Bei dieser Strategieentwicklung spielen Ärztinnen und Ärzte eine zentrale Rolle.

Im Zeitalter der vielfach geforderten Selbstverantwortung von Patientinnen und Patienten kann aber auch die Ansicht vertreten werden, dass diese die Möglichkeit haben sollten, pharmakogenetische Tests selber zu erwerben und anzuwenden. Der Nuffield-Report meint hierzu: Sofern pharmakogenetische Tests bezüglich Wirksamkeit und Nebenwirkungen von Medikamenten klare Aussagen machen, spricht nichts dagegen, den Test direkt in der Apotheke - mit oder ohne ärztliches Zeugnis - oder via Internet zu 
erwerben und ohne Fachhilfe durchzuführen [3]. Allerdings muss dann sichergestellt werden, dass Patientinnen und Patienten leichten $\mathrm{Zu}$ gang zu umfassenden und ausgewogenen Informationen haben und geschulte Fachpersonen als Auskunftspersonen zur Verfügung stehen.

\section{Arzt-Patienten-Verhältnis}

Befürworter der Pharmakogenetik und -genomik stellen in Aussicht, künftig «massgeschneidert» behandeln zu können. Durch diese Versprechen für eine «individualisierte Therapie» oder «personalisierte Medizin» könnten Patientinnen und Patienten eine grosse Erwartungshaltung an die Erfolgswahrscheinlichkeit der Therapie entwickeln. Wenn Erwartungshaltung und Realität für die Patientinnen und Patienten zu weit auseinanderdriften, wird deren Vertrauen in die behandelnde Person beeinträchtigt. Weiter kann die zunehmende Komplexität der zu berücksichtigenden Einflussfaktoren die Patientinnen und Patienten verunsichern und eine therapeutische Entscheidung erschweren.

Auf der anderen Seite können sich verbesserte Behandlungsmöglichkeiten in der Praxis auf das Arzt-Patienten-Verhältnis positiv auswirken: Wirksamere Behandlungen oder weniger Nebenwirkungen werden das Vertrauen der Patienten in die fachliche Kompetenz ihres Arztes stärken. Als Folge ist mit einer besseren Compliance auf seiten der Patienten zu rechnen. Auch die Bindung zwischen Arzt und Patient wird damit gestärkt. Eine bessere Compliance und eine engere Arzt-Patienten-Beziehung können wiederum einem allfälligen Ärztetourismus entgegenwirken. Als Folge sind Kosteneinsparungen zu erwarten.

\section{Neue Herausforderungen an die gemeinsame Entscheidungsfindung}

Die Interpretation pharmakogenetischer Testresultate oder der Entscheidungsprozess für oder gegen einen pharmakogenetischen Test oder ein genomikbasiertes Medikament bei ausgewählten Zielgruppen stellt wegen des komplexen Zusammenspiels genetischer Faktoren mit nicht-genetischen Einflüssen hohe Anforderungen an die behandelnden Ärztinnen und Ärzte [4, 5]. Mit der Verbreitung von pharmakogenomischen Tests und Medikamenten im medizinischen Alltag ist daher auch damit zu rechnen, dass der Konsultationsprozess zwischen Leistungserbringer und Patienten noch umfangreicher und an- spruchsvoller wird. Besonders die Vermittlung von Wahrscheinlichkeitsvorhersagen wird an die Kommunikation zwischen Anbietern, Leistungserbringern (Arzt/Apotheke) und Patientinnen und Patienten neue Anforderungen stellen [6].

Zudem dürften sich Arzt und Patient mit neuen Fragestellungen konfrontiert sehen, nämlich dann wenn:

- ein Test zusätzliche Informationen bezüglich einer Krankheitsprädisposition enthält, die zugleich auch Auswirkungen auf andere Familienmitglieder haben. Therapieüberlegungen können zu einer zunehmenden Auseinandersetzung mit Fragen des familiären Hintergrundes einer Erkrankung führen. So weiss man zum Beispiel, dass es eine genetisch definierte Untergruppe von Alzheimererkrankungen gibt. Für deren Behandlung steht ein Medikament zur Verfügung, welches das Fortschreiten der Krankheit verlangsamt. Ein Gentest im Vorfeld der Abklärungen für die spezifische Behandlung einer erkrankten Patientin kann deshalb zugleich Auswirkungen auf Familienmitglieder haben, analog zur klassischen genetischen Prädispositionstestung.

- das Testresultat zeigt, dass es für die getesteten Patientinnen und Patienten kein geeignetes Medikament gibt. Angenommen, ein genetischer Tests hat ergeben, dass sich Patientinnen und Patienten für ein bestimmtes Medikament grob in zwei Gruppen einteilen lassen: Bei Gruppe A wirkt das Medikament mit 80\% Wahrscheinlichkeit, bei Gruppe B mit nur 15\% Wahrscheinlichkeit. Zeigt ein Testresultat nun, dass eine Person mit grosser Wahrscheinlichkeit zur Gruppe B gehört und eine Therapie nur mit 15\% Wahrscheinlichkeit ansprechen wird, stellt sich die Frage, ob dennoch ein Therapieversuch gemacht werden soll. Es ist evident, dass die Frage im Einzelfall je nach Schweregrad der Erkrankung sowie den bestehenden Optionen anderer Behandlungen und dem Risiko für schwere Nebenwirkungen anders zu beantworten ist. Damit verbunden werden aber auch Fragen der Kostenübernahme für Therapien mit nur mässigem Therapieerfolg relevant werden. Beispielsweise zeigen die Erfahrungen im Umgang mit Herceptin in Deutschland, dass eine Therapie mit Herceptin nur dann von den Krankenkassen übernommen wird, wenn eine Frau mit Brustkrebs im Vorfeld mittels eines pharmakogenetischen Tests als «Responder» eingestuft wurde.

Dabei ist wichtig hervorzuheben, dass Wahrscheinlichkeits- und Kostenaspekte schon 
heute die Entscheidungsfindung erschweren, mit der Implementierung von Pharmakogenetik und -genomik aber akzentuiert werden. Die grössere Aufteilung von Patienten in Untergruppen führt parallel dazu auch zu einer Vereinfachung der Entscheidungsfindung. Die relative Bedeutung dieser Aspekte hängt sehr vom Schweregrad der Erkrankung, vom Schweregrad allfälliger Nebenwirkungen, von der Verfügbarkeit paralleler Therapiemöglichkeiten sowie der Einstellung des Patienten und Arztes ab.

\section{Schlussfolgerungen}

Um die Vorteile von pharmakogenetischen Tests und genomikbasierten Medikamenten für die Arzt-Patienten-Beziehung optimal nutzen $\mathrm{zu}$ können, müssen die Akteure im Bereich Therapieverschreibung und -beratung auf ihre neuen Aufgaben vorbereitet werden. Dazu zählen beispielsweise gezielte Aus- und Weiterbildungsmassnahmen der Leistungserbringer zur Genomik und Pharmakogenomik sowie zur Kommunikation von Wahrscheinlichkeiten.

Eine wichtige Zielgruppe für die Vermittlung dieser Beratungsaspekte werden die Grundversorger sein. Ihnen sollte für die Patientinnen und Patienten einfach verständliches Informationsmaterial zur Verfügung gestellt werden. Es ist darauf zu achten, dass die Informationen ausgewogen und umfassend sind und mögliche Interessen der Industrie transparent dargelegt werden. Des weiteren müssen Massnahmen getroffen und Strukturen geschaffen werden, um daraus entstehende zusätzliche Belastungen im ohnehin schon zeitintensiven Ärztealltag rechtzeitig abzufangen (zum Beispiel Entwicklung neuer Beratungsmodelle, adäquate finanzielle Entschädigung von Beratungen, etc.).

\section{Literatur}

1 Schmid M, Wang J für das Schweizer «Future Patient» Forschungsteam. Der Patient der Zukunft: Das Arzt-Patienten-Verhältnis im Umbruch. Schweiz Ärztezeitung 2003;84(41):2133-5.

2 Human Genetics Commission. Inside Information: Balancing interests in the use of personal genetic information. London: Department of Health; 2002.

3 Nuffield Council on Bioethics. Pharmacogenetics, 2003.

4 Abbott A. With your genes? Take one of these, three times a day. Nature 2003;425:760-2.

5 Haga SB, Khoury MJ, Burke W. Genomic profiling to promote a healthy lifestyle: not ready for prime time. Nature Genetics 2003;34:347-50.

6 Bolt LLE, van Delden JJM, Kalis A, Derijks HJ, Leufkens HGM. Tailor-made pharmacotherapy: future developments and ethical challenges in the field of pharmacogenomics. Utrecht: CBG; 2002. 